\title{
An Occlusion Reasoning Scheme for Monocular Pedestrian Tracking in Dynamic Scenes
}

\author{
Sourav Garg and Swagat Kumar \\ Innovation Lab \\ Tata Consultancy Services \\ New Delhi, India 201301 \\ Email: \{swagat.kumar,garg.sourav\}@tcs.com
}

\author{
Rajesh Ratnakaram and Prithwijit Guha \\ Department of Electronics and Electrical Engineering \\ Indian Institute of Technology Guwahati \\ Guwahati, Assam, India 781039 \\ Email: \{pguha,ratnakaram\}@iitg.ernet.in
}

\begin{abstract}
This paper looks into the problem of pedestrian tracking using a monocular, potentially moving, uncalibrated camera. The pedestrians are located in each frame using a standard human detector, which are then tracked in subsequent frames. This is a challenging problem as one has to deal with complex situations like changing background, partial or full occlusion and camera motion. In order to carry out successful tracking, it is necessary to resolve associations between the detected windows in the current frame with those obtained from the previous frame. Compared to methods that use temporal windows incorporating past as well as future information, we attempt to make decision on a frame-by-frame basis. An occlusion reasoning scheme is proposed to resolve the association problem between a pair of consecutive frames by using an affinity matrix that defines the closeness between a pair of windows and then, uses a binary integer programming to obtain unique association between them. A second stage of verification based on SURF matching is used to deal with those cases where the above optimization scheme might yield wrong associations. The efficacy of the approach is demonstrated through experiments on several standard pedestrian datasets.
\end{abstract}

\section{INTRODUCTION}

In this paper, we look into the problem of tracking multiple targets using a monocular, possibly moving, uncalibrated camera. It has several applications in areas like smart vehicles, robotics and video surveillance. It can be used for extracting higher level of information from a video, such as, event detection, crowd analysis etc. The task involves locating concerned targets, assigning unique IDs to each one of them and generating trajectories for them. The problem is challenging as one has to deal with several complex situations like changing background, camera motion, wide variation in appearance and illumination and, partial or full occlusion.

One of the popular approach is to use tracking-by-detection framework which has become one of the popular approach to solve this problem. In this framework, a detector is used to locate targets in each frame and then associate these detections across frames. This approach however suffers from the limitations of the object detector which may yield false positives and missing detections. On the other hand, resolving associations between detected targets across frames may become challenging under conditions of group formation and occlusions for long duration.

In most of the methods, the data association problem is solved by optimizing the detection assignments over a temporal window [1] [2] [3]. Nevatia's group, particularly, focused on hierarchical association at multiple levels [2] [4] [5] where the tracklets are associated to form longer trajectories. The association is formulated as a MAP problem which is solved using the Hungarian algorithm. These were mostly off-line approaches where the frames were revisited over multiple iterations. In their latest work [6], authors learn a Conditional Random Field (CRF) model to learn appearance and motion model that also takes into account relative positions between the targets. There are other approaches that make use of particle filter to solve the tracking problem as in [7] [8].

In this paper, we re-look at the multi-target tracking problem with a focus on simplifying the entire approach. We primarily focus on using monocular camera images in contrast to other methods that use stereo-vision system [9] [10], laser scanner [11], night vision [12] or LIDAR [13] [14], sometimes in addition to vision. We aim to make online decisions on a frame-by-frame basis unlike other approaches where a temporal window is used for incorporating future information for resolving association in the current frame [6]. Such methods are prone to frequent ID switches and trajectory fragmentation due to noisy and ambiguous observation [5]. We attempt to overcome these limitations of a frame-by-frame approach in this paper. We use the standard JRoG detector as used by the authors in [6] as we are primarily making comparison with their results. However, any other object detector could be used locating pedestrians in the video. Readers may refer to [15] [16] [17] for a survey on the state of the art methods in pedestrian detection. Once a new person is detected in a frame, a colour-based mean-shift tracker is initialized. This mean-shift (MS) tracker [18] combined with a Kalman Filter (KF) [19] based motion predictor is used to localize this target in the new frame. In order to carry out successful tracking, it is necessary to resolve association between the currently detected target windows with those estimated from the previous frame using KF and MS tracker. This is challenging as the windows may overlap with each other resulting in many-to-one or oneto-many associations. The need for an occlusion reasoning scheme in a multi-agent tracking problem is illustrated in Figure 1. It is shown that the agent IDs get interchanged during occlusion when the associations are not properly and hence, there is a need for having an effective occlusion reasoning scheme.

Our main contribution lies in proposing an occlusion reasoning scheme (ORS) that uses an affinity matrix and binary 


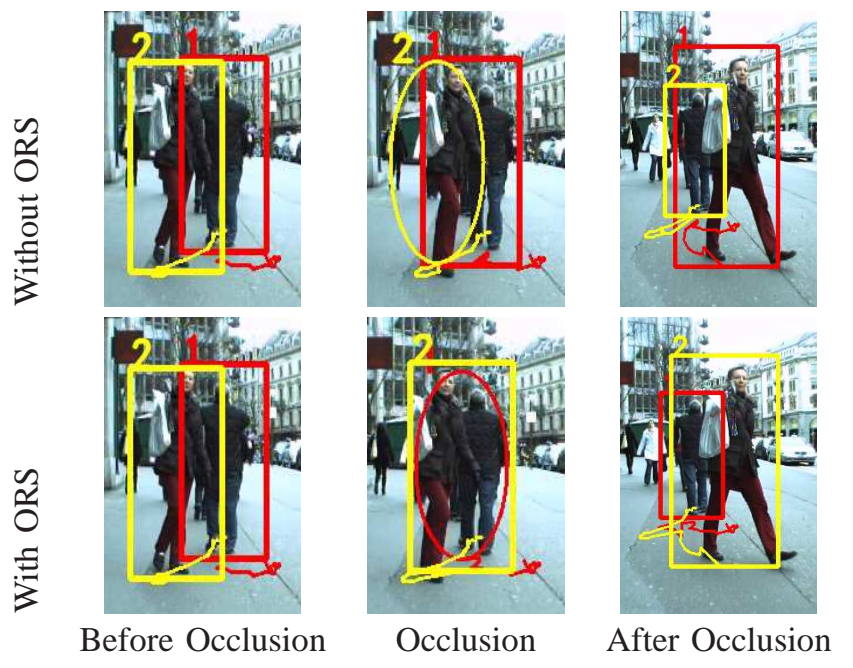

Fig. 1: Need for Occlusion Reasoning Scheme (ORS) in multiagent tracking. Without ORS, agent IDs are interchanged between the overlapping agents 1 and 2. This ID switch is prevented using ORS.

integer programming to resolve the data association problem between a pair of frames. The affinity matrix represents the 'closeness' between a pair of tracking windows. The binary integer programming (BIP) module returns unique associations between the agents of this pair. Since the resolution is based on some feature based scalar value function in the affinity matrix, the resulting association might still be incorrect in some extreme cases. We use agent pairing information from the last frame and SURF matching to provide a second stage of verification over the decision obtained from the BIP module. The resulting algorithm is tested on several datasets and the performance is compared with the state of the art.

It is to be noted that one can use Hungarian algorithm [1] [6] in place of BIP for resolving associations. However, we envisage that BIP or linear programming would allow us to incorporate constraints which are not confined to be the elements of a matrix, as is the case with the Hungarian algorithm. One such use case is demonstrated in [20]. However, in the present scenario, Hungarian algorithm is found to be computationally more efficient compared to BIP in obtaining same solution.

Even though, it is an initial work with a lot of scope for improvement, we believe that the material presented in this paper would provide a lot of useful insights which can be appreciated by the readers. The rest of this paper is organized as follows. The proposed method is provided in Section [I] The analysis of experimental results is provided in Section III followed by conclusion in Section IV

\section{PROPOSED APPROACH}

In order to explain our approach, we would use the following notations. A given video sequence is represented by the symbol $I_{k}, k=1,2, \cdots, N$ indicating that the video has a total of $N$ frames. As stated earlier, any standard human detector is used to locate pedestrians in each frame.

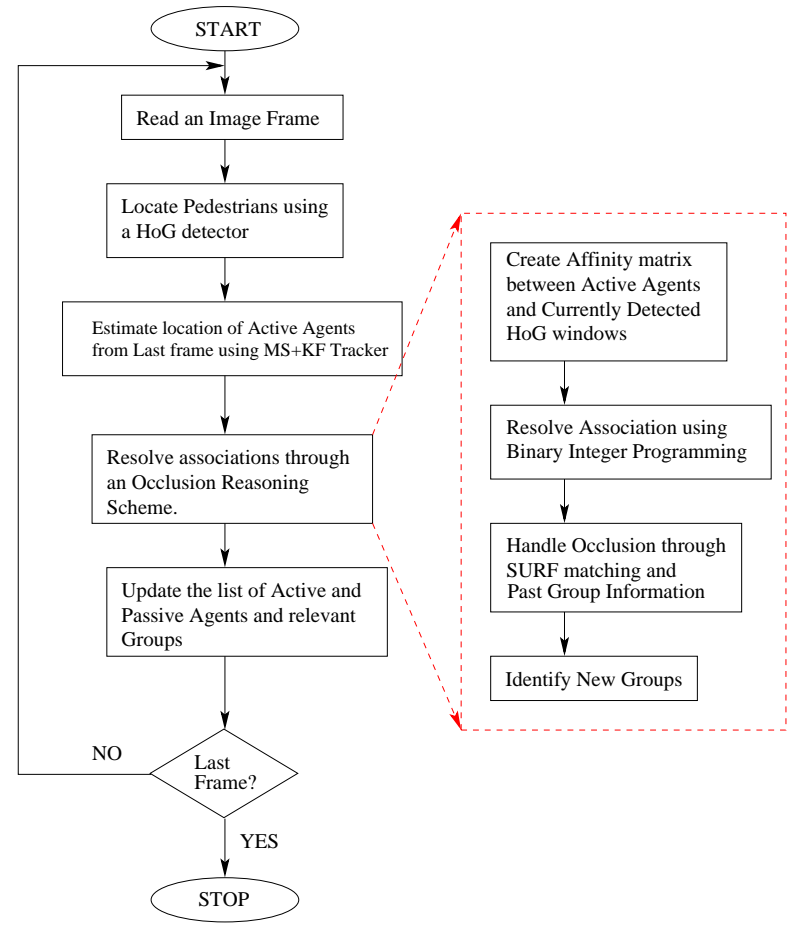

Fig. 2: Outline of our approach for pedestrian tracking. The proposed occlusion reasoning scheme is shown in the red box.

Each person detected or tracked is called an agent which is represented by a bounding box (BB) surrounding the person and is labeled with a global ID. The agents for a given frame $I_{k}$ is represented by the symbol $A_{k}^{i}, i=1,2, \cdots, n$, where $n$ is the number of agents that are found by the detector in the frame.

The proposed method for carrying out pedestrian detection and tracking is shown as a flowchart in Figure 2, The method consists of four steps. The first step involves applying a human detector to locate pedestrians in each frame. The second step involves estimating the location of agents from the last frame using a tracker that uses mean-shift algorithm [18] and a Kalman Filter [19]. It is necessary to associate these agents from the last frame with those obtained from the detector in the current frame. The association simply means assigning appropriate labels or IDs to the currently detected target windows. The problem becomes difficult when the agents come together to form groups or undergo partial or full occlusion. We propose an occlusion reasoning scheme to solve this association problem between the past agents and the currently detected target windows. This reasoning scheme is explained next in this section. Once the associations are resolved, the list agents is updated by adding new agents which are found in the current frame. A list for the pairs of agents which overlap with each other is also maintained which is also essential for dealing with the cases of occlusion.

\section{A. Occlusion reasoning scheme}

The occlusion reasoning scheme includes four main steps. The first step involves creating an affinity matrix between the estimated agent windows obtained from the last frame (using 
tracker) and the persons detected in the current frame by the detector. This matrix is utilized in the next step to resolve association between these two groups of agents using binary integer programming. The resulting associations may contain few errors arising out of difficult cases like occlusion. Hence a second stage of verification based on SURF matching and pairing information from the last frame. Once the associations with the previous agents are resolved, the newly detected agents are given new agent IDs. The details for each of these steps are provided below.

1) Affinity Matrix: An illustration of an affinity matrix for a given frame is shown in Figure 3 Let us assume that the number of agents found by the detector in the current frame $I_{k}$ is $n$ while the number of agents obtained from the last frame $\left(I_{k-1}\right)$ is $m$. The location of these agents from the last frame is estimated using $\mathrm{KF}+\mathrm{MS}$ tracker. These estimated agents are represented by the symbol $\hat{A}_{k-1}^{k}$. Hence the affinity matrix $S_{k}$ for this frame has a dimension $m \times n$ with each element having a value obtained from a scalar function given by:

$$
S_{k}(i, j)=f(O, B C)=\alpha_{1} O(i, j)+\alpha_{2} B C(i, j)
$$

where $O(i, j)$ is the percentage overlap between the two bounding boxes given by

$$
O(i, j)=\frac{\hat{A}_{k-1}^{k}(i) \cap A_{k}(j)}{\hat{A}_{k-1}^{k}(i) \cup A_{k}(j)}
$$

and $B C(i, j)$ is the Bhattacharya Coefficient computed between the corresponding bounding boxes representing similarity based on histogram matching. The weights $\alpha_{1}$ and $\alpha_{2}$ are normalized weights which are decided a priori indicating the relative importance of individual factors in the overall function. The values of the matrix elements $S_{k}(i, j)$ lie between 0 and 1,0 being no overlap or similarity and 1 indicating high level of affinity or similarity between the windows. This affinity matrix indicates the 'closeness' between a pair of windows. This matrix is used in the next step to resolve associations between the agents obtained from the previous frame and the persons detected in the current frame.

2) Resolving associations using Binary Integer Programming (BIP): The association of currently detected target windows with those obtained from the last frame is not straight forward. This is due to the fact that this association depends on multiple features. The association obtained using one feature might conflict with that obtained using another feature. Secondly, there might be cases of one-to-many or many-to-one associations between the two sets of windows. The first cause is alleviated to some extent by forming the affinity matrix where multiple features or criteria are combined to form a unique scalar function that indicates the similarity or affinity between a pair of windows. The second problem is solved by posing it as an optimization problem which is solved by using binary integer programming [21]. The elements of affinity matrix are considered to be the decision variables and constraints are put over the rows and columns of the matrix, so that many-to-one or one-to-many associations do not occur. We use the COIN-OR CBC [22] library in order to solve this problem. The parameters of the proposed BIP formulation are as follows:

- $\quad S_{k}(i, j) \in \mathbb{R}$ : coefficient of matching or similarity between a given pair of windows in the affinity matrix.
Agents found in the current frame using the HoG detector

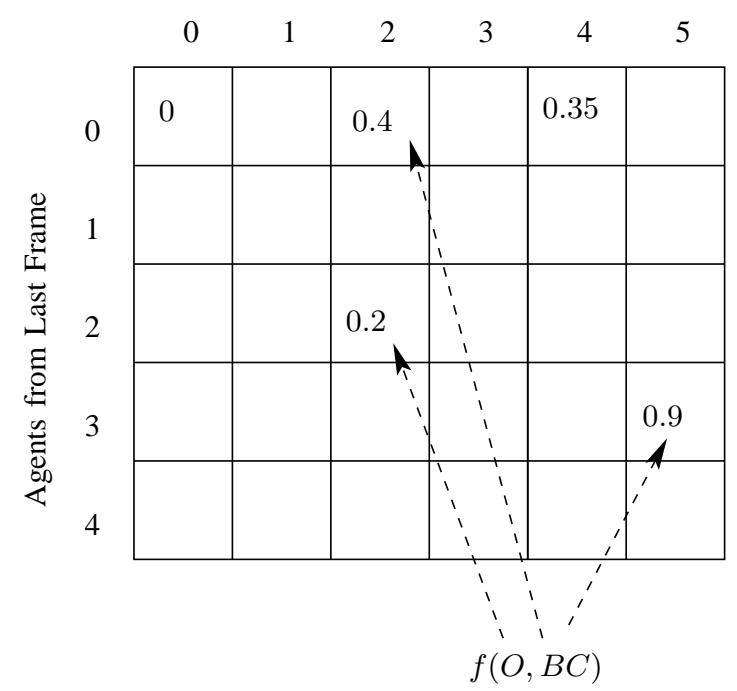

Fig. 3: Affinity Matrix for a given frame. A non-zero value indicates 'closeness' between a pair of windows. The values are normalized between 0 and 1 .

- $u_{i, j} \in\{0,1\}:$ decision variable, $u_{i, j}=1$, if the matching pair $(i, j)$ is selected, else $u_{i, j}=0$.

The optimization problem is now stated as follows:

$$
\begin{array}{lll}
\arg \max _{u} & \sum_{i=1}^{m} \sum_{j=1}^{n} S_{k}(i, j) u_{i, j} & \\
\text { subject to } & \sum_{i=1}^{m} u_{i, j} \leq 1 & \forall j \\
& \sum_{j=1}^{n} u_{i, j} \leq 1 & \forall i \\
& u_{i, j} \in\{0,1\} & \forall i, j
\end{array}
$$

The objective function (3a) aims at maximizing the association between a pair of windows as given by the affinity matrix. The constraints (3b) and (3c) allow only one-to-one association between the considered pair of windows. The bound $3 \mathrm{~d}$ restricts the decision variable to be binary. The decision variables having value of 1 in the BIP solution correspond to the selected pair of windows. The optimization process for resolving association is illustrated in Figure 4 We consider frame number 91 in the ETH2 dataset. In this image, three detected target windows are labelled as $a, b$ and $c$ respectively. From the previous frame, 4 windows are obtained using KF+MS tracker. These windows have labels 3,4,5 and 6. The conflicting associations arise due to the pairs shown in green ellipse in the affinity matrix. The BIP module gives rise to a binary matrix providing unique associations between the two sets of windows. Hence, the target window $a$ is assigned the label $5, b$ is assigned 4 and $c$ is assigned label 3. The window ID 6 is not associated with any target window and hence appears as an ellipse in the final image. 
HoG detected windows

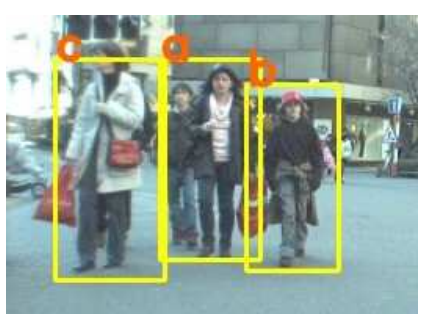

Frame \#91

Dataset: ETH2

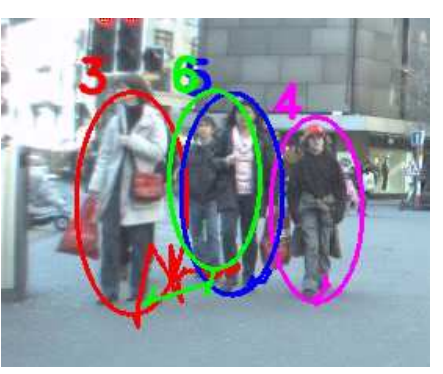

Agents obtained from $\mathrm{KF}+\mathrm{MS}$ Tracker

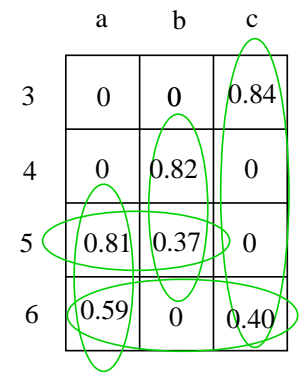

Affinity Matrix
Binary

Integer

Programming

Labelled HoG Windows
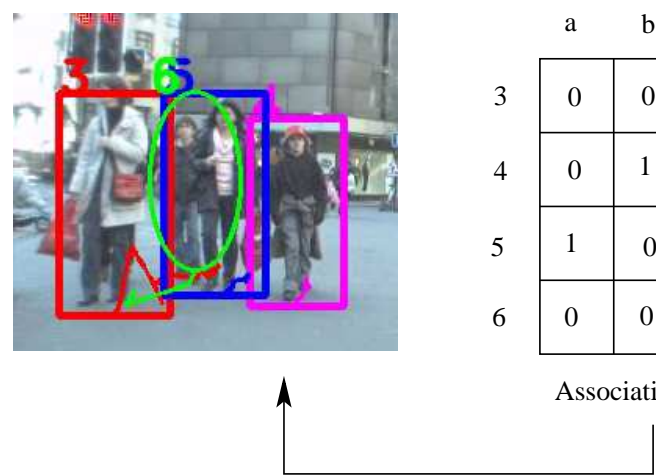

Fig. 4: Resolving associations using Binary Integer Programming. The HoG detected windows $(a, b, c)$ are associated with the agents obtained from the tracker $(3,4,5,6)$. Conflicting associations are shown as green ellipse in the affinity matrix. The output of BIP is a binary association matrix providing final labels for the HoG windows. Agent 6 is not associated with any HoG window.

3) Occlusion Handling through SURF Matching: We will use some additional notations in order to explain the method presented in this section. As stated above, each new agent is assigned an unique global ID which is used to identify this agent wherever it is visible in a video. We use the notation $L\left(A_{k}(i)\right)$ to denote the global ID of a given agent in the frame $I_{k}$. The symbol $\left\{L\left(A_{k}\right)\right\}$ refers to the set of labels for all active agents in this frame. We also define a set $G_{k}$ that consists of all pairs of agent IDs that overlap with each other. In other words,

$$
G_{k} \triangleq\left\{g_{k}^{i}\right\}, i=1,2, \cdots, r
$$

where each element $g_{k}^{i}$ is a pair of agent labels (IDs) given by

$$
g_{k}^{i}=\left\{L\left(A_{k}(p)\right), L\left(A_{k}(q)\right)\right\}, p \neq q,(p, q) \in\{0,1, \ldots, n\}
$$

As explained in the previous section, the global labels of the active agents obtained from the detector in the current frame is resolved by the binary integer programming (BIP) module which uniquely assigns the labels of agents from the last frame $L\left(A_{k-1}\right)$ to the currently detected agents. Let us denote this set of labels for the currently detected agents by the symbol $\left\{L^{-}\left(A_{k}\right)\right\}$. Some of the labels obtained from the BIP module might be erroneous, particularly for those agents which get occluded or appear in groups in the current frame. This is due to the fact that the decision of the BIP module solely depends on the features used in the affinity matrix. Even though multiple features or cues will provide robustness, yet it can guarantee correct decisions for all cases.

Therefore, a second stage of verification is employed to correct these labels by using the pairing information obtained from the last frame $G_{k-1}$ and SURF matching as explained in Algorithm 11. In this algorithm, $n\left(G_{k-1}\right)$ refers to the cardinality of the set $G_{k-1}$. The basic idea is that if one of the agents in the pair disappears in the current frame, SURF matching is used to recognize the agent which is available, and is assigned the corresponding agent label. The new set of global IDs obtained for the currently active agents is denoted by $\left\{L\left(A_{k}\right)\right\}$. Once the labels are found, a new set of agent pairs are found based on whether they overlap or not. This group is denoted by $G_{k}$ and will be utilized in the next iteration. The scheme is explained pictorially in Figure 5 Let us assume that the agents $(A, B)$ form a pair in the previous frame $I_{k-1}$ and in the current frame only one window is detected by the detector. Let us call it $C$. Also assume that the BIP module associates window $C$ with $A$. In this case, the SURF matching between the pair $(A, C)$ and $(B, C)$ is used to confirm the final association. The one with maximum percentage match is selected as the correct association pair.

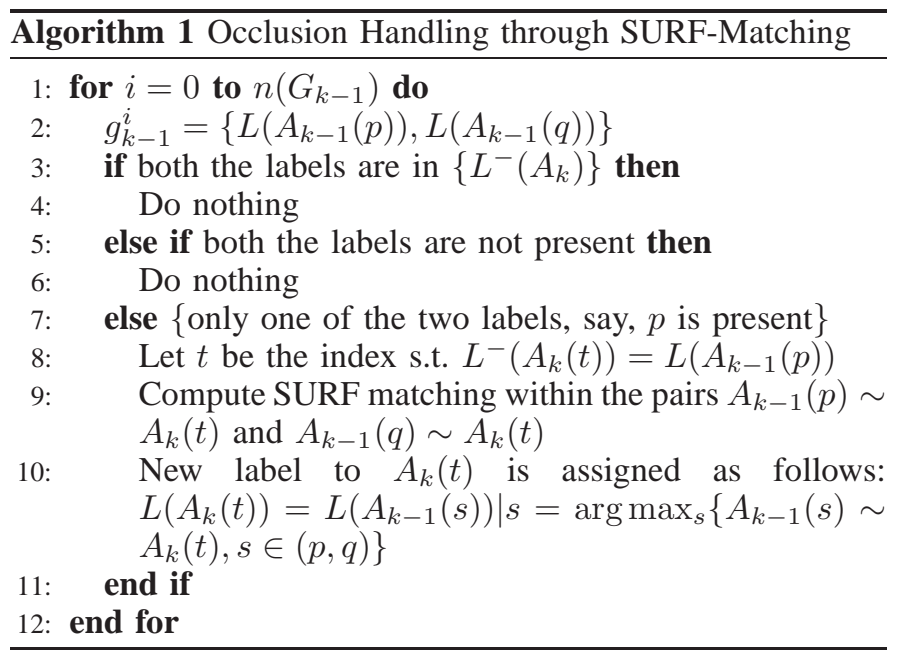

\section{B. Estimating agent location using Kalman Filter and Mean- shift tracker}

As stated earlier, the location of agents from the last frame is estimated in the current frame using a mean-shift tracker 


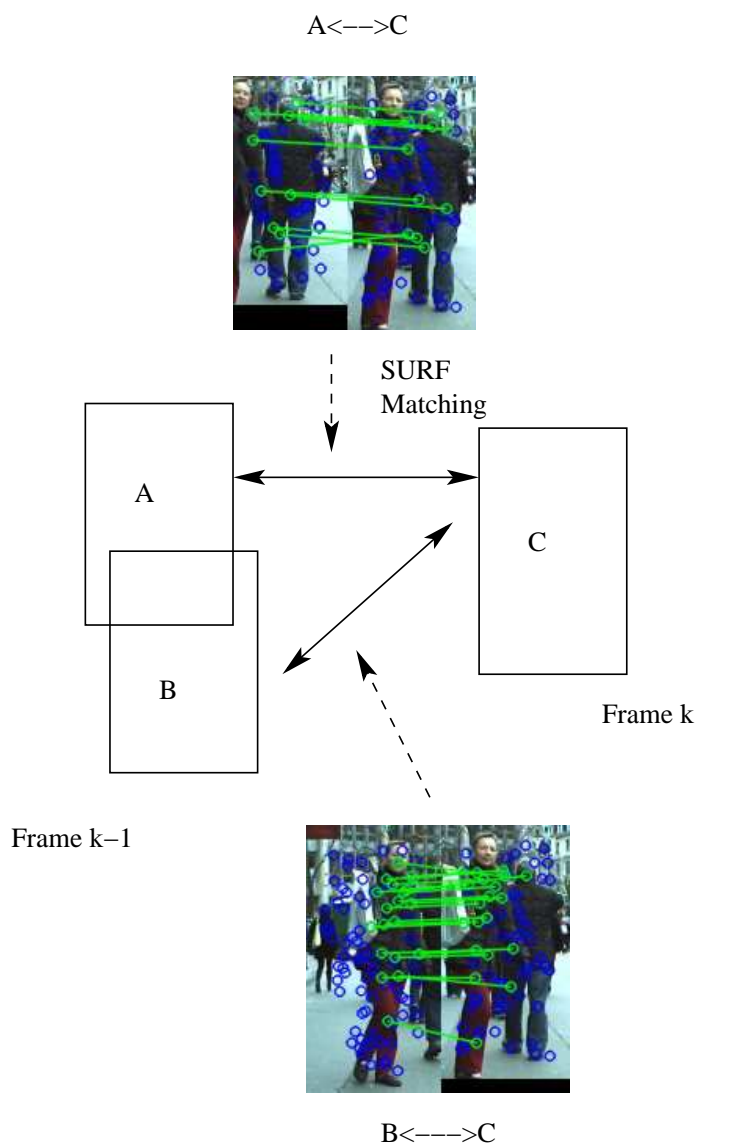

Fig. 5: Occlusion Handling through SURF Matching. If one of the agents from the previous pairs is not present in the current frame, use SURF-matching to identify the available agent.

combined with a Kalman Filter. It is well known that the detector may not provide detection for a given agent in every frame where it is located. In case of detection failure, a Kalman Filter could be used to predict its location. The Kalman Filter itself learns from the observations obtained from the detector. The reliance on object detector which is computationally expensive could be reduced by using a mean-shift tracker [18] that uses a colour histogram to locate target in the next frame. The mean-shift tracker is initialized for each new agent obtained from the detector. In cases where the detector fails to locate this agent, the Kalman Filter and the mean-shift tracker could be used together to confirm the location of the said agent. Moreover, use of mean-shift tracker along with a Kalman Filter could be used to reduce the computational cost by reducing the search area for the detector.

\section{EXPERIMENTAL RESULTS}

The performance of our algorithm is evaluated on three datasets which are the same as used by Yang and Nevatia [6]: TUD dataset [23], PETS 2009 [24] and ETH dataset [9]. Since we wanted to compare our results with those reported in [6], we used the same detector and performance parameters to compute our tracking results. The resulting comparison is provided in Table I The performance parameters used are: precision, recall, false alarm per frame (FAF), ground truth
(GT), mostly tracked trajectories (MT), partially tracked trajectories (PT), mostly lost trajectories (ML), number of trajectory fragmentation (Frag) and number of id switches (IDS). Please refer to the above paper for definitions of various parameters mentioned in this table. Few such parameters computed for one of the ETH datasets are shown in Figure 7 In this figure, false trajectories (FT) are those which are generated due to a false detection made by the HoG detector. Some of the snapshots of various agent trajectories for different datasets are shown in Figure 6. The complete tracking video is made available on web [25] for the convenience of readers. The snapshots show some of the cases where our scheme is able to resolve associations resulting in accurate tracking for the agent.

We can see that the performance of our algorithm is not good compared to the Nevatia's latest work [6] even though we have better tracking performance such MT, PT and ML. It is to be noted that Nevatia's work is based on tracklets that introduces latency into the decision making process unlike our approach where we take decision per frame basis. However, this is an initial work which can be improved in several ways. Some of them are as follows:

1) We have more trajectory fragmentation and IDS because, we create new IDs for the same agent if it remains occluded or not detected for a certain number of frames. One approach would be to compare the currently detected targets with not only with the last frame but also past trajectories. 2) We are using Kalman Filter as the motion predictor for each agent. Probably, this assumption is not valid in case of camera motion. The relative position of agents could be used as a parameter for resolving associations between agents as suggested in [6]. 3) The direction of each agent's motion along with motion coherence can be incorporated into the affinity matrix. 4) The values of $\alpha_{i}$ in equation (1) is decided a priori by the user. This could be treated as variables to be optimized over another set of constraints. 5) Taking cue from Nevatia's work [6], the relative location of pedestrians could be utilized to compensate for camera motion.

\section{CONCLUSION AND FUtURE WORK}

In this paper, we take a relook at the multi-target tracking problem. Our main contribution lies in proposing an occlusion reasoning scheme to solve the association among the detected agents on a frame-by-frame basis. The scheme defines an affinity matrix that depicts the closeness between the estimated agent windows of the previous frame with those obtained from a detector in the current frame. This affinity matrix is later used by a binary integer programming (BIP) module to find unique associations between these pair of windows. A second stage of verification based on SURF-matching is employed to deal with the wrong associations generated by the BIP module. This module makes use of past agent pair information to resolve the agent identities in the current frame. The performance of our algorithm is compared with the latest work in this field. It is still an initial work with a lot of scope for improvement. The work presented here will be useful for students and practicing engineers who would like to understand the process and the underlying challenges of the problem. 


\begin{tabular}{|c|c|c|c|c|c|c|c|c|c|}
\hline Method & Recall (\%) & Precision (\%) & $\overline{F A F}$ & GT & MT $(\%)$ & PT (\%) & ML (\%) & Frag & IDS \\
\hline \multicolumn{10}{|c|}{ ETH Dataset } \\
\hline$\overline{\text { Our approach }}$ & $\overline{87.7}$ & $\overline{49.8}$ & $\overline{6.76}$ & $\overline{125}$ & $\overline{78.2}$ & $\overline{17.8}$ & $\overline{4.0}$ & 182 & $\overline{27}$ \\
\hline Yang \& Nevatia (2014) & 79.0 & 90.4 & 0.637 & 125 & 68.0 & 24.8 & 7.2 & 19 & 11 \\
\hline \multicolumn{10}{|c|}{ PETS 2009 Dataset } \\
\hline Our approach & $\overline{97.3}$ & $\overline{\overline{68.0}}$ & 2.66 & 19 & $\overline{94.7}$ & $\overline{5.3}$ & $\overline{0.0}$ & $\overline{64}$ & $\overline{8}$ \\
\hline Yang \& Nevatia (2014) & 93.0 & 95.3 & 0.268 & 19 & 89.5 & 10.5 & 0.0 & 13 & 0 \\
\hline \multicolumn{10}{|c|}{ TUD Dataset } \\
\hline Our approach & $\overline{94.2}$ & $\overline{77.0}$ & 1.74 & 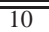 & $\overline{100}$ & $\overline{0.0}$ & $\overline{0.0}$ & $\overline{10}$ & $\overline{3}$ \\
\hline Yang \& Nevatia (2014) & 87.0 & 96.7 & 0.184 & 10 & 70.0 & 30.0 & 0.0 & 1 & 0 \\
\hline
\end{tabular}

TABLE I: Comparison of Tracking results for different datasets

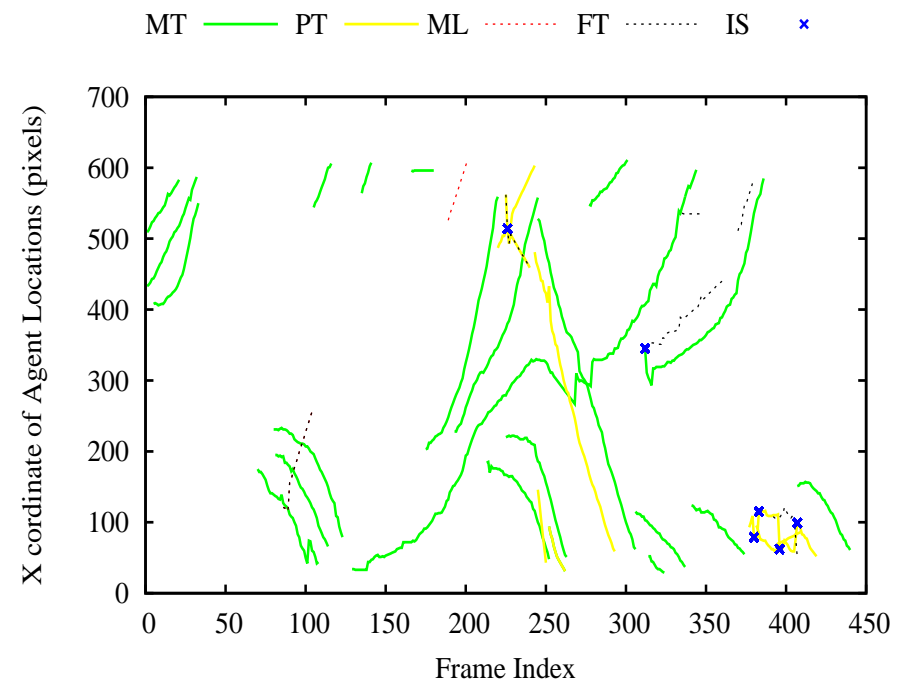

Fig. 7: Tracking performance for ETH2 dataset. False trajectories are generated due to wrong detections by the HoG detector. Most agents (shown in green) are correctly tracked with the help of the proposed occlusion reasoning scheme.

\section{REFERENCES}

[1] A. A. Perera, C. Srinivas, A. Hoogs, G. Brooksby, and W. Hu, "Multiobject tracking through simultaneous long occlusions and split-merge conditions," in Computer Vision and Pattern Recognition, 2006 IEEE Computer Society Conference on, vol. 1. IEEE, 2006, pp. 666-673.

[2] B. Wu and R. Nevatia, "Detection and tracking of multiple, partially occluded humans by bayesian combination of edgelet based part detectors," International Journal of Computer Vision, vol. 75, no. 2, pp. 247-266, 2007.

[3] M. Andriluka, S. Roth, and B. Schiele, "People-tracking-by-detection and people-detection-by-tracking," in Computer Vision and Pattern Recognition, 2008. CVPR 2008. IEEE Conference on. IEEE, 2008, pp. $1-8$.

[4] C. Huang, B. Wu, and R. Nevatia, "Robust object tracking by hierarchical association of detection responses," in Computer Vision-ECCV 2008. Springer, 2008, pp. 788-801.

[5] Y. Li, C. Huang, and R. Nevatia, "Learning to associate: Hybridboosted multi-target tracker for crowded scene," in Computer Vision and Pattern Recognition, 2009. CVPR 2009. IEEE Conference on. IEEE, 2009, pp. 2953-2960.

[6] B. Yang and R. Nevatia, "Multi-target tracking by online learning a crf model of appearance and motion patterns," International Journal of Computer Vision, vol. 107, no. 2, pp. 203-217, 2014.

[7] K. Okuma, A. Taleghani, N. De Freitas, J. J. Little, and D. G. Lowe, "A boosted particle filter: Multitarget detection and tracking," in Computer Vision-ECCV 2004. Springer, 2004, pp. 28-39.
[8] M. D. Breitenstein, F. Reichlin, B. Leibe, E. Koller-Meier, and L. Van Gool, "Robust tracking-by-detection using a detector confidence particle filter," in Computer Vision, 2009 IEEE 12th International Conference on. IEEE, 2009, pp. 1515-1522.

[9] A. Ess, B. Leibe, K. Schindler, and L. Van Gool, "A mobile vision system for robust multi-person tracking," in Computer Vision and Pattern Recognition, 2008. CVPR 2008. IEEE Conference on. IEEE, 2008, pp. 1-8.

[10] C. G. Keller, M. Enzweiler, M. Rohrbach, D. Fernandez Llorca, C. Schnorr, and D. M. Gavrila, "The benefits of dense stereo for pedestrian detection," Intelligent Transportation Systems, IEEE Transactions on, vol. 12, no. 4, pp. 1096-1106, 2011.

[11] K. C. Fuerstenberg, K. C. Dietmayer, and V. Willhoeft, "Pedestrian recognition in urban traffic using a vehicle based multilayer laserscanner," in Intelligent Vehicle Symposium, 2002. IEEE, vol. 1. IEEE, 2002, pp. 31-35.

[12] F. Xu, X. Liu, and K. Fujimura, "Pedestrian detection and tracking with night vision," Intelligent Transportation Systems, IEEE Transactions on, vol. 6 , no. 1, pp. 63-71, 2005.

[13] M. Szarvas, U. Sakai, and J. Ogata, "Real-time pedestrian detection using lidar and convolutional neural networks," in Intelligent Vehicles Symposium, 2006 IEEE. IEEE, 2006, pp. 213-218.

[14] C. Premebida, G. Monteiro, U. Nunes, and P. Peixoto, "A lidar and vision-based approach for pedestrian and vehicle detection and tracking," in Intelligent Transportation Systems Conference, 2007. ITSC 2007. IEEE. IEEE, 2007, pp. 1044-1049.

[15] M. Enzweiler and D. M. Gavrila, "Monocular pedestrian detection: Survey and experiments," Pattern Analysis and Machine Intelligence, IEEE Transactions on, vol. 31, no. 12, pp. 2179-2195, 2009.

[16] D. Geronimo, A. M. Lopez, A. D. Sappa, and T. Graf, "Survey of pedestrian detection for advanced driver assistance systems," Pattern Analysis and Machine Intelligence, IEEE Transactions on, vol. 32, no. 7, pp. 1239-1258, 2010.

[17] P. Dollar, C. Wojek, B. Schiele, and P. Perona, "Pedestrian detection: An evaluation of the state of the art," Pattern Analysis and Machine Intelligence, IEEE Transactions on, vol. 34, no. 4, pp. 743-761, 2012.

[18] D. Comaniciu, V. Ramesh, and P. Meer, "Kernel-based object tracking," IEEE Transactions on Pattern Analysis and Machine Intelligence, vol. 25, no. 4, pp. 1-14, April 2003.

[19] S. Haykin, Adaptive Filter Theory, 4th ed. Pearson Education, 2009.

[20] C. Park, T. J. Woehl, J. E. Evans, and N. D. Browning, "Minimum cost multi-way data association for optimizing multitarget tracking of interacting objects," 2014.

[21] G. L. Nemhauser and L. A. Wolsey, Integer and combinatorial optimization. Wiley New York, 1988, vol. 18 .

[22] J. Forrest and R. Lougee-Heimer, "Cbc user guide," INFORMS Tutorials in Operations Research, pp. 257-277, 2005.

[23] A. Andriyenko, S. Roth, and K. Schindler, "An analytical formulation of global occlusion reasoning for multi-target tracking," in Computer Vision Workshops (ICCV Workshops), 2011 IEEE International Conference on. IEEE, 2011, pp. 1839-1846.

[24] "Pets 2009 dataset," http://ftp.pets.rdg.ac.uk/pub/PETS2009/Crowd_PETS09_data

[25] R. Rajesh, "Experimental video for pedestrian tracking," http://youtu.be/cfIjqOMTD1I 


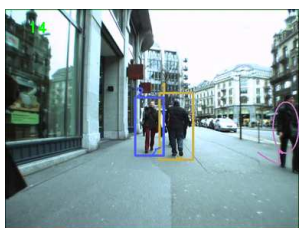

(1a)

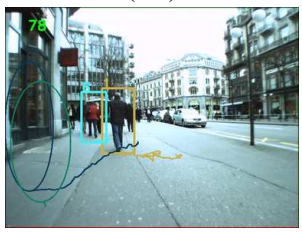

(1f)

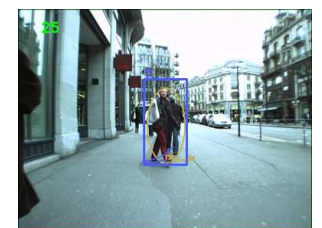

(1b)

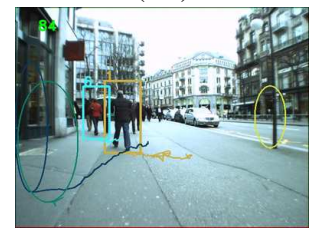

$(1 \mathrm{~g})$

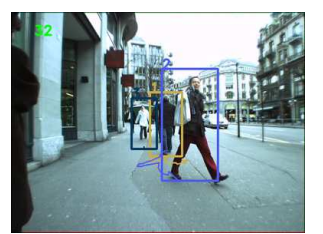

(1c)

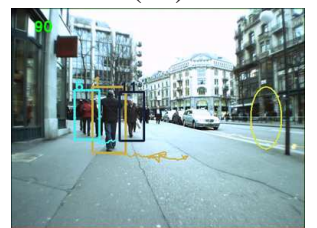

(1h)

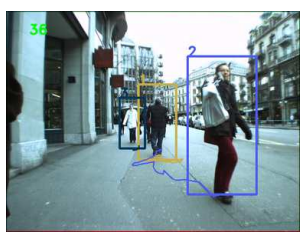

(1d)

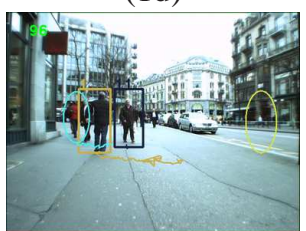

(1i)

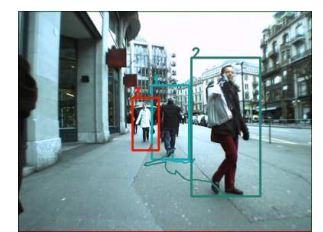

(1e)

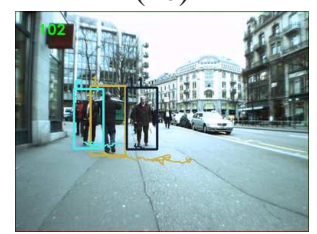

$(1 \mathrm{j})$

ETH1 dataset: (1a-1e) Trajectory of agent 2 (lady on left with blue window) intersects with that of agent 1 (person in the centre) without any ID switch. (1f-1j): Agent 1 (brown) is tracked successfully even when it forms group with other agents. However ID switch occurs with agent 6 (cyan).

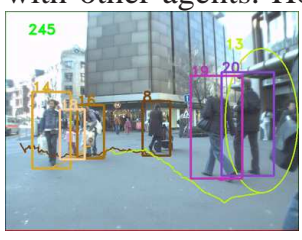

$(2 \mathrm{a})$

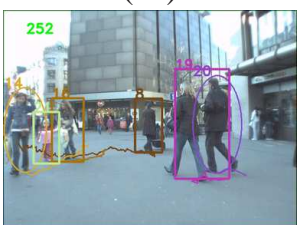

(2f)

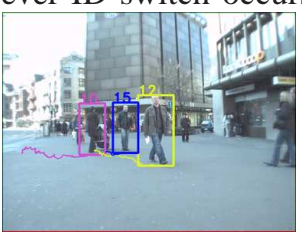

(2b)

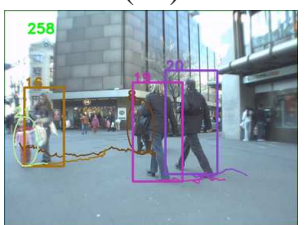

$(2 \mathrm{~g})$

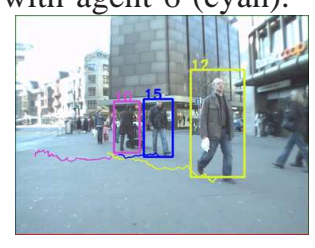

$(2 \mathrm{c})$

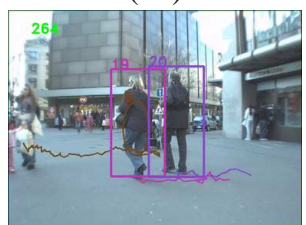

(2h)

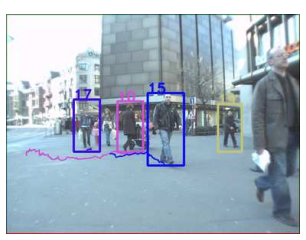

$(2 \mathrm{~d})$

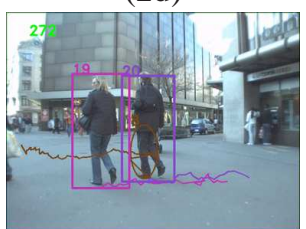

(2i)

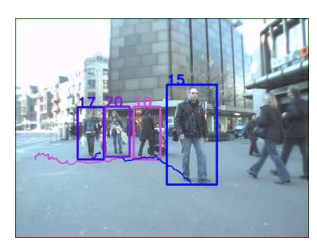

$(2 \mathrm{e})$

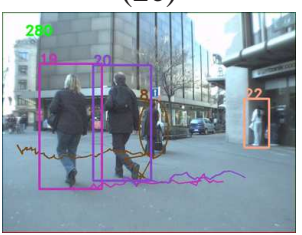

$(2 \mathrm{j})$

Dataset ETH2: (2a-2e) Agent 10 (lady in pink box) is tracked successfully through the crowd in spite of several instances of occlusion. (2f-2j) All the agents are tracked successfully inspite of grouping and occlusion.

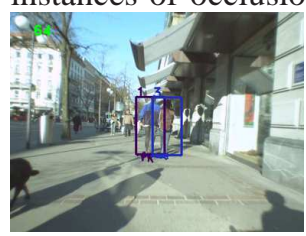

(3a)

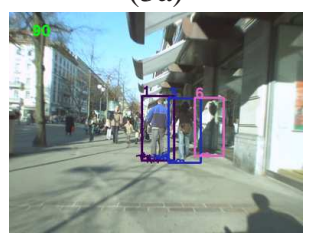

(3f)

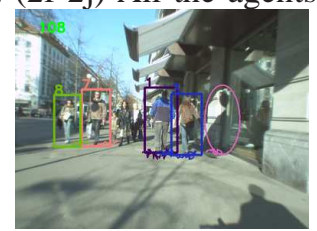

(3b)

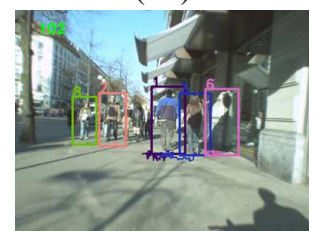

(3g)

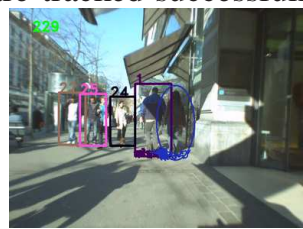

(3c)

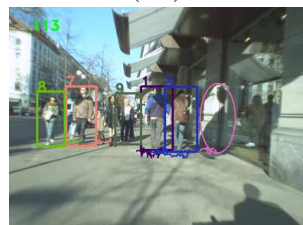

(3h)

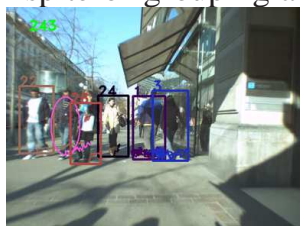

(3d)

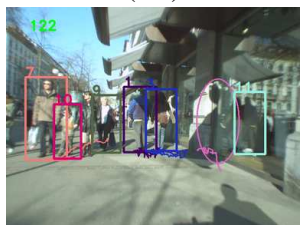

(3g)

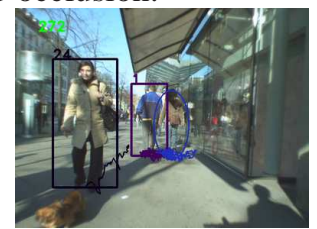

(3e)

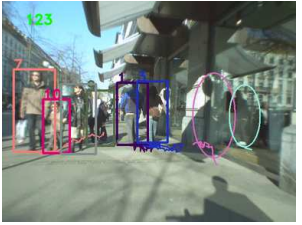

(3i)

ETH3: (3a-3e) Agents 1 and 3 are tracked successfully in presence of various false positives due to shadow and reflection. (3f-3j) Shows two false trajectories generated as a result of detection failure (agent 6 and 11).

Fig. 6: Snapshots of trajectories generated for various agents for three different ETH datasets. Predicted agent location is shown as an ellipse. Each detected agent window is shown with a rectangular bounding box with its agent ID. It shows several instances where the ID switch is prevented and the target is tracked successfully despite occlusion and other effects. 


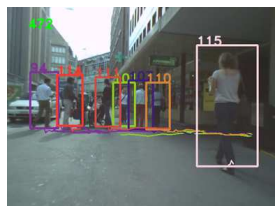

$(4 a)$

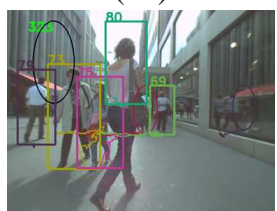

(4f)

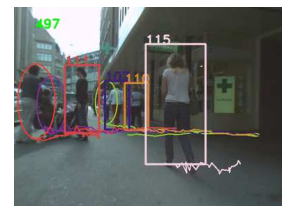

(4b)

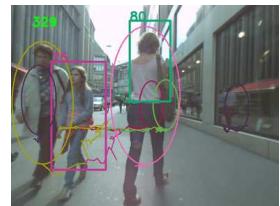

$(4 \mathrm{~g})$

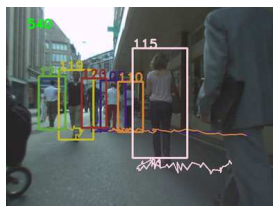

$(4 c)$

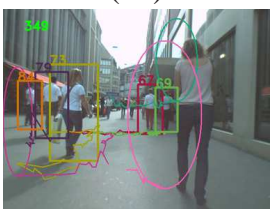

(4h)

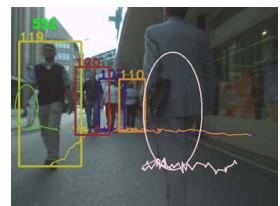

$(4 d)$

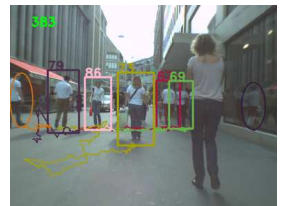

(4i)

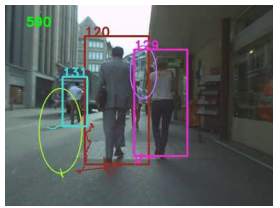

$(4 \mathrm{e})$

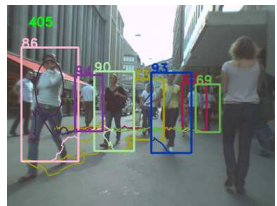

$(4 \mathrm{j})$

ETH4: (4a-4e) The lady on right gets a new ID as it recovers from occlusion. It also shows several cases of ID switch. (4f-4j) Agent 79 (navy blue) is tracked successfully over a span of more than 100 frames and then undergoes an ID switch. This video has significant camera motion which resulting in poor tracking performance

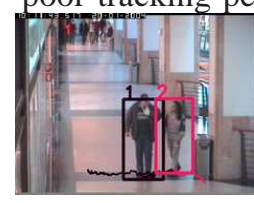

$(5 \mathrm{a})$

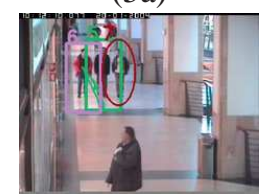

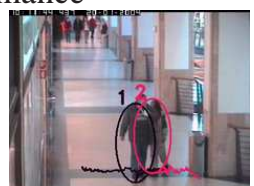

$(5 b)$

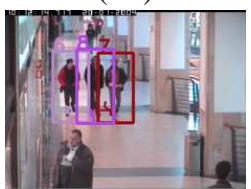

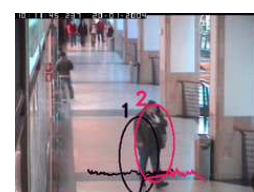

$(5 \mathrm{c})$

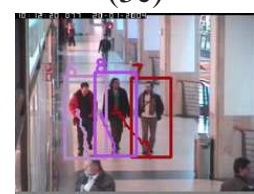

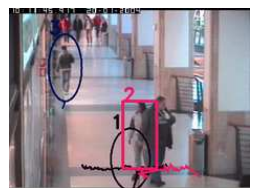

$(5 \mathrm{~d})$

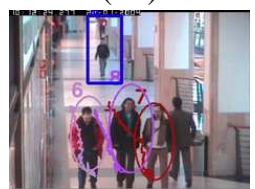

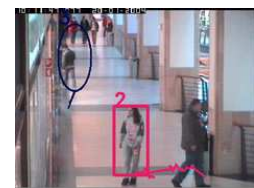

$(5 \mathrm{e})$

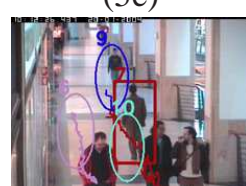

CAVIAR1: (5a-5e) Identity of agent 2 (shown in pink) is restored once it recovers from occlusion by agent 1. (5f-5j) Two ID switch occurs among the three agents which move in a group.

Fig. 6: Snapshots of tracking performance for Video datasets ETH4 and CAVIAR1. CAVIAR dataset has a static background while ETH datasets have dynamic background. 